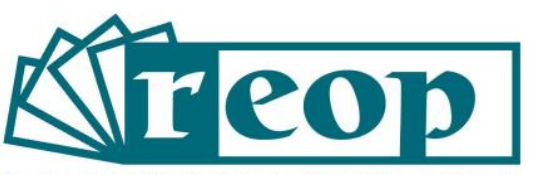

Revista Española de Orientación y Psicopedagogía

\title{
IDENTIFICACIÓN DE LOS FACTORES PRINCIPALES ASOCIADOS A LA ELECCIÓN DE GRADOS UNIVERSITARIOS EN EL ÁMBITO AGROALIMENTARIO
}

\section{IDENTIFICATION OF THE MAIN FACTORS ASSOCIATED WITH THE ELECTION OF UNIVERSITY DEGREES IN THE AGRO-FOOD FIELD}

\author{
Cristina López-Cózar Navarro ${ }^{1}$ \\ Sonia Benito-Hernández \\ Universidad Politécnica de Madrid. Escuela Técnica Superior de Ingeniería Agronómica, \\ Alimentaria y de Biosistemas. Departamento de Economía Agraria, Estadística y Gestión de \\ Empresas. Madrid, España \\ Tiziana Priede-Bergamini \\ Universidad Europea de Madrid. Facultad de Ciencias Sociales. Departamento de Economía y \\ Empresa. Madrid, España
}

\section{RESUMEN}

La industria agroalimentaria es considerada un sector económico estratégico, por lo que la vocación, preparación y capacitación de sus profesionales se convierte en una cuestión clave para cualquier país. Sin embargo, en los últimos 20 años, estamos asistiendo a un decremento de las tasas de jóvenes matriculados en la educación superior en las áreas de ciencia y tecnología en general, y especialmente, en carreras de la rama agroalimentaria. Por este motivo, el trabajo que se presenta se centra en identificar los principales factores que influyen en la decisión del alumnado al elegir los grados de las ingenierías y ciencias en este campo particular. Para ello, se ha realizado una encuesta personal a una muestra de 430 estudiantes que han elegido estas titulaciones en la Universidad Politécnica de Madrid. A partir de la información recabada, un análisis factorial ha permitido detectar cuatro factores relevantes en la elección del grado en el

${ }^{1}$ Correspondencia: Cristina López-Cózar Navarro. Correo-e: cristina.lopezcozar@upm.es, web: $\underline{\text { http://www.upm.es }}$ 
ámbito agroalimentario: la procedencia (lugar de residencia y tipología del centro escolar) del estudiante, el momento de la decisión en el que el alumnado elige el grado que quiere cursar (antes o durante el bachillerato, antes o después de la EBAU), el itinerario académico previo (tecnológica, salud, sociales y artes) y sus motivaciones personales (vocacional, tradición familiar, afinidad o descarte). Este trabajo pretende esclarecer los factores que influyen en la elección de esta carrera universitaria con el fin de ayudar a orientadores e investigadores académicos, así como a psicopedagogos como apoyo en su función.

Palabras clave: Elección profesional, enseñanza superior, ámbito agroalimentario, orientación universitaria, motivación.

\section{ABSTRACT}

The agro-food industry is considered a strategic economic sector, so the aptitude, preparation and training of its professionals becomes a key issue for any country. However, in the last 20 years, we are witnessing a decrease in the rates of young people enrolling in higher education areas of science and technology in general, and especially, in the agro-food industry degrees. Consequently, this paper aims to analyze the main factors that influence students when choosing the degrees of engineering and science but in this particular field. With this purpose, a personal survey was used on a sample of 430 students who had chosen these degrees at the Polytechnic University of Madrid. Based on the collected information, a factorial analysis allowed the detection of four relevant factors in the choice of the agro-food field degree: origin of the student (place of residence and type of school); the moment of the decision in which the student chooses the future degree (during or after high school, before or after EBAU), the previous academic itinerary (technological, health, social sciences or arts); and their personal motivations (vocational, family tradition, affinity or by discard). This research aims to clarify the factors that influence the choice of this specific higher education degree in order to help academic counselors and researchers, as well as psycho-pedagogues to support their function.

Key Words: Professional choice, higher education, agro-food field, university guidance, motivation.

\section{Cómo citar este artículo:}

López-Cózar, C., Benito, S. y Priede, T. (2020). Identificación de los factores principales asociados a la elección de grados universitarios en el ámbito agroalimentario. Revista Española de Orientación y Psicopedagogía, 31(3), 26-44. https://doi.org/10.5944/reop.vol.31.num.3.2020.29260

\section{Introducción}

El decremento de las tasas de estudiantes, especialmente mujeres, matriculados en las titulaciones superiores de las ramas de ciencia y tecnología es causa de preocupación desde hace 
años, sobre todo, por el impacto que tienen estas áreas en el desarrollo económico y social de los países (Comisión Europea, 2004). Efectivamente, trabajos como los de Fensham (2004), Vázquez-Alonso y Manassero-Mas (2015) y Vázquez Romero y Blanco-Blanco (2019) muestran el desinterés de la juventud hacia carreras técnicas. Por ello, revertir esta situación se ha convertido en un gran reto tanto nacional como internacional (Vázquez-Alonso y Manassero-Mas, 2015).

Este declive se ha visto acentuado de forma alarmante en el número de preinscripciones y matrículas en estudios del área agroalimentaria. El sector agroalimentario es considerado estratégico y clave en cualquier país, de igual o mayor trascendencia que el farmacéutico o el energético, pues es el que alimenta a toda su población. Por lo tanto, la vocación, la formación y la capacitación de sus profesionales resulta de gran interés. Así pues, el presente trabajo se centra en analizar la primera cuestión planteada, estudiando la motivación y el contexto en el que el alumnado toma la decisión sobre el grado universitario que va a cursar.

Es ampliamente reconocida la relevancia que tiene la elección de la titulación (Martínez, Castro, Zurita y Lucena, 2015; Caldera, Reynoso, González y Zamora, 2018). Los motivos por los cuales una persona elige qué grado estudiar generan unas expectativas que, posteriormente, serán confrontadas constantemente en el transcurso de la carrera, por lo que constituye un elemento importante del abandono académico (Freixa, Llanes y Venceslao, 2018; Tuero, Cervero, Esteban y Bernardo, 2018). En este sentido, Domínguez, Álvarez-Pérez y López-Aguilar (2012) ponen de manifiesto la necesidad de mejorar la orientación que recibe el alumnado al respecto y, al mismo tiempo, señala al docente como la persona idónea para ofrecer la información académica y asesorar sobre las diversas salidas profesionales a su alcance. Igualmente, ÁlvarezPérez, López-Aguilar y Pérez-Jorge (2015), Bernardo Gutiérrez et al. (2016), Álvarez-Pérez y López-Aguilar (2019) y Rodríguez-Muñiz, Areces, Suárez-Álvarez, Cueli y Muñiz (2019) también muestran la importancia de ayudar a cada estudiante a definir su proyecto formativo, dado que, en muchos casos, esta elección se realiza cuando se es demasiado joven y no se tiene una idea clara de las metas que persiguen, ni de la proyección futura de las carreras elegidas. Por ello, es pertinente profundizar en el conocimiento de las variables que influyen en dicha decisión en el momento de acceder a la enseñanza superior.

Así pues, el objetivo de este trabajo es identificar los factores que determinan la elección de las titulaciones en el área agroalimentaria como primera o segunda opción en la preinscripción realizada antes de formalizar la matrícula universitaria. La identificación de estos factores puede esclarecer y ayudar a orientadores y docentes, tanto de la educación en general, como la del sector agroalimentario en particular, a conocer cuáles son las motivaciones del alumnado de secundaria a la hora de decidirse por este tipo de carreras y, por tanto, ayudar en su labor con el fin de reducir las tasas de abandono y fracaso universitario. El ámbito de estudio de la investigación se limita a estudiantes que actualmente están cursando titulaciones de grado en la Universidad Politécnica de Madrid (UPM); en particular, en la Escuela Técnica Superior de Ingeniería Agronómica, Alimentaria y de Biosistemas (ETSIAAB). Las carreras ofertadas por la ETSIAAB objeto de análisis son: Grado en Ingeniería y Ciencia Agronómica (ICA), Grado en Ingeniería Alimentaria (IA), Grado en Ingeniería Agroambiental (IAMB), Grado en Ciencias Agrarias y Bioeconomía (BIOECO), Grado en Tecnología de las Industrias Agrarias y Alimentarias (IND), y Grado en Ingeniería Agrícola, plan 2017 (IAGR17) y Grado en Ingeniería Agrícola, plan 2010 (IAGR10).

Para conseguir este objetivo se presenta, en primer lugar, una revisión de la literatura mostrando los antecedentes teóricos y empíricos publicados más relevantes. A continuación, se describe la elección de la muestra, la técnica utilizada, la construcción de variables y la metodología de análisis del trabajo empírico. Posteriormente, se exponen los resultados estadísticos obtenidos, para finalizar mostrando las principales conclusiones, implicaciones y limitaciones de las evidencias encontradas. 


\section{Antecedentes y fundamentación teórica}

La literatura previa ha puesto de manifiesto la trascendencia que tiene la elección de los estudios superiores para lograr un adecuado desarrollo académico y profesional de la persona egresada (Martínez et al., 2015; Caldera et al., 2018). En efecto, se puede considerar que el momento en el que se elige la titulación universitaria es determinante en la vida del estudiante y, además, presenta consecuencias posteriores en la trayectoria del individuo (Corrales et al., 2020). Rivas (1990) plantea que cuando los jóvenes llevan a cabo este trascendental proceso de toma de decisión, suelen tener muy poca experiencia y falta de madurez; enfocan la decisión como algo puntual e intrascendente, y se ven afectados tanto por factores internos -en menor grado- como por elementos externos. Esto implica que estas decisiones, en ocasiones, suelen ser poco reflexivas, improvisadas y muy condicionadas por variables externas, lo cual, como es lógico, presenta consecuencias posteriores.

Sánchez (2001), en una investigación sobre las circunstancias de elección de los estudios superiores y las necesidades de orientación académica, señala que, al elegir la formación universitaria, cada estudiante se enfrenta a la definición de un proyecto profesional y, por tanto, un proyecto de vida. Apunta que se trata de una elección muy personal que le afecta y le compromete, por lo que tener una motivación individual fuerte hacia el grado elegido, así como la capacidad para tomar tal decisión, se consideran aspectos esenciales para evitar el fracaso.

Como señalan Bethencourt et al. (2008), el rendimiento universitario está directamente relacionado, entre otros elementos, con el grado de satisfacción con la titulación elegida. Autores como Álvarez-Pérez et al. (2015) y Álvarez-Pérez y López-Aguilar (2019) apuntan que quienes acceden a la universidad con información adecuada sobre las opciones académicas, teniendo una idea clara de sus propios intereses y expectativas, tendrán más facilidades para lograr una correcta promoción en sus estudios. Por el contrario, quienes eligen en el último momento, no tienen claro qué carrera cursar, o se matriculan en un grado que no sea de su preferencia, estarán más abocados a problemas de rendimiento e insatisfacción, llevando incluso al abandono en su primer año (Tuero et al., 2018).

Pablo-Lerchundi, Núñez-del-Río y González-Tirados (2014), en su trabajo sobre la relación entre la motivación sobre la elección de distintas carreras técnicas y el desarrollo profesional, encuentran que altos niveles de motivación intrínseca del alumnado conducen a una mayor productividad y un mayor nivel de satisfacción en el ámbito laboral. Madden, Webber, Ford y Crowder (2018) muestran que existe una relación directa entre las asignaturas preferidas en la educación secundaria y la elección del grado universitario. Así mismo, Kinnunen et al. (2018), a partir de una encuesta realizada con una muestra de estudiantes de Reino Unido, Suecia y Finlandia, muestran cómo influyen las expectativas en las titulaciones elegidas, si bien, también señalan la importancia de gestionar dichas expectativas. Por su parte, Doña y Luque (2019) muestran cómo las motivaciones de tipo intrínsecas, especialmente la vocación, logran niveles superiores de satisfacción con los estudios elegidos. En la misma línea, Corrales, Moreno, Sánchez y Zamora (2020), con una muestra de estudiantes de bachillerato, encuentran que prevalecen las variables de motivación intrínseca sobre las de la motivación extrínseca; si bien, señalan que esta diferencia es menor en el caso del alumnado de ciencias y tecnología.

Respecto a los factores asociados a la elección de los estudios universitarios, en la literatura previa se pueden encontrar diferentes trabajos que analizan esta cuestión de forma concreta, empleando diversos enfoques y técnicas. De una forma general, se puede afirmar que el alumnado suele elegir la carrera que más le gusta siempre que sea posible cursarla en la localidad 
en la que vive (Rivas 1990; Mosteiro y Porto 2000). Fernández, Peña, Viñuela, y Torío (2007) encuentran las preferencias personales como el elemento más influyente en la decisión sobre qué estudiar, mientras que la valoración que otorga el alumnado a los procesos de orientación en los centros educativos es relativamente baja, situándose por debajo de la opinión de su familia. Martínez-Martínez et al. (2017) señalan el gusto por la profesión como el factor más determinante al elegir un grado universitario. Por su parte, trabajos como los de Pablo-Lerchundi et al. (2014) y Rodríguez-Muñiz et al. (2019) muestran que hay que considerar dos grandes grupos de motivaciones: intrínsecas, aquellas relacionadas con los gustos y preferencias individuales, y extrínsecas o circunstancias externas, tales como salidas laborales o asesoramiento recibido por parte de familiares o docentes.

Algunos trabajos han buscado distintos perfiles motivacionales en función del género y el tipo de carrera, encontrándose, en algunas ocasiones, diferencias importantes en este sentido (Doña y Luque, 2019). Para el caso del género, esto implica que los motivos que influyen en la elección de las enseñanzas universitarias son diferentes para los hombres que para las mujeres (Gámez y Marrero, 2003; Vieira, Monteiro, Carrieri, Guerra y Brant, 2019). Navarro y Casero (2012) muestran, a través de una investigación transversal, que la elección de las mujeres suele encaminarse hacia carreras de humanidades, ciencias sociales, experimentales, jurídicas y relativas a la salud, siendo las razones principales el gusto personal, la vocación y la necesidad de ayudar a los demás; sin embargo, los hombres suelen decantarse por carreras de tipo técnico con el objetivo de alcanzar un buen salario. También García Guevara (2002) muestra la existencia de factores, desde culturales e individuales hasta otros como el proceso de globalización de la economía y el avance tecnológico, que amplían la brecha entre las mujeres y los hombres que se matriculan en grados de ingeniería (civiles, industriales e informáticas, sobre todo). Martínez et al. (2015) encuentran que las mujeres prefieren grados de ciencias de la salud y ciencias sociales, mientras que los hombres se decantan por titulaciones más técnicas (arquitectura e ingeniería). No obstante, otras investigaciones no han encontrado diferencias estadísticamente significativas que hagan variar los motivos de la elección de estudios superiores en cuanto al género (González 2005, Mosteiro y Porto 2000).

Por otro lado, la motivación a la hora de tomar la decisión también parece variar con la carrera elegida. Sánchez (2001) clasifica los motivos influyentes en personales, profesionales y sociofamiliares, Ilevando a cabo un análisis para una muestra compuesta por estudiantes de diversas titulaciones de las universidades madrileñas. Esta autora concluye, de forma general, que la vocación y la empleabilidad son los factores que más influyen en la elección. Ahora bien, los motivos profesionales son más relevantes para los grados de humanidades, mientras que las influencias sociofamiliares son más importantes para los estudios técnicos, sociales y jurídicos. Se reconoce, por tanto, la existencia de una relación entre el tipo de carrera cursada y las causas que determinan la decisión. En la misma línea, los trabajos de Boe (2012) y de Vázquez-Alonso y Manassero-Mas (2015) muestran que, entre los factores determinantes para la elección de carreras técnicas, destacan el interés del alumnado por los temas científicos, la relación con profesores de ciencias, determinados programas de televisión y las expectativas de empleo, encuentran que el alumnado de carreras de ciencias e ingenierías no tiene una motivación clara a la hora de elegir sus estudios

Finalmente, también existen otros trabajos que desarrollan el análisis desde una perspectiva diferente. Así, Solsona et al. (2016) se centran en el estudio de los factores que determinan la elección de la universidad, encontrando que los más determinantes son la proximidad geográfica, la calidad ocupacional, el itinerario cursado, la reputación de la propia universidad, las notas de corte y el nivel educativo de la madre. Por su parte, Rego-Agraso y Rial-Sánchez (2017) también señalan la proximidad geográfica junto con la salida profesional y la vocación, como determinantes de la elección de titulación, si bien, en este caso, se refiere a estudiantes de formación profesional, tanto de grado medio como superior. 


\section{Método}

\section{Muestra y procedencia de los datos}

La población objeto de estudio de la que se ha extraído la muestra es el número de personas matriculadas en titulaciones de grado en la ETSIAAB durante el curso 2017-2018 (tabla 1). En la literatura previa existen otros trabajos realizados para un entorno determinado, por ejemplo, la investigación de Fernández et al. (2007) se desarrolla en el Principado de Asturias, Martínez et al. (2015) en la provincia de Granada, la de Solsona et al. (2016) en la universidad de Lleida o la de Rego-Agraso y Rial-Sánchez (2017) en Galicia.

Dado que el tipo de población es finita (1.210 estudiantes) el cálculo del tamaño muestral (n) se utilizará la siguiente fórmula (1):

$$
n=\frac{N * Z_{\mathrm{x}}^{2} * p * q}{d^{2} *(N-1)+Z_{\alpha}^{2} * p * q}
$$

A partir de dicha ecuación, el tamaño muestral necesario es de 430 sujetos. En la muestra inicial se consiguieron 441 respuestas, de las cuales se eliminaron aquellas que procedían de estudiantes de programas internacionales y permanecerían en la Escuela un periodo de tiempo inferior a un año, así como todas las respuestas incoherentes. Tras ser depurados los datos, se quedaron en 430 , es decir, se obtuvo una tasa de respuesta del $36,44 \%$. La muestra a su vez es representativa de la población a estudiar, tal y como puede observarse en la tabla 1.

\section{Tabla 1}

Representatividad de la muestra

\begin{tabular}{lcccc}
\hline Grado & No estudiantes & $\%$ & Frecuencia & $\%$ \\
\hline ICA & 276 & 22,80 & 86 & 20,0 \\
\hline IA & 371 & 30,66 & 110 & 25,5 \\
\hline IAMB & 144 & 11,90 & 51 & 11,8 \\
\hline BIOECO & 42 & 3,47 & 24 & 5,6 \\
\hline IND & 127 & 10,49 & 64 & 8,8 \\
\hline IAGR17 & 87 & 7,19 & 36 & 13,7 \\
\hline IAGR10 & 163 & 13,47 & 59 & 100,0 \\
\hline Total & 1.210 & 100 & 430 & \\
\hline
\end{tabular}

Fuente: Elaboración propia

La técnica empleada para la recopilación de información ha sido la encuesta personal, en línea con otros trabajos previos como el de Gámez y Marrero (2003), Bethencourt et al. (2008), Rego-Agraso y Rial-Sánchez (2017) o Freixa et al. (2018). Dicha encuesta se realizó físicamente en las aulas, pasando por todos los cursos de cada uno de los grados. Así pues, el método de muestreo es aleatorio (quien asistía a clase el día de la encuesta). Como instrumento se utilizó un cuestionario, en el cual las preguntas se formularon con un lenguaje claro para evitar problemas 
de interpretación, y cuya duración final se estimó inferior a diez minutos, para de esta manera, favorecer la cooperación tanto del encuestado, como del docente que en ese momento se encontrara dando clase.

\section{Definición de las variables objeto de estudio}

La tabla 2 recoge el conjunto de variables de la encuesta realizada. Se detalla brevemente su definición y los valores que adoptan.

\section{Tabla 2}

Definición de variables

\begin{tabular}{|c|c|c|c|c|}
\hline Variable & $\begin{array}{l}\text { Tipo de } \\
\text { Variable }\end{array}$ & Definición & Nombre & Valores \\
\hline Prioridad & Dependiente & $\begin{array}{l}\text { Orden que asignó a la carrera que está } \\
\text { cursando en la preinscripción que realizó } \\
\text { para matricularse en la Universidad }\end{array}$ & Prioridad & $\begin{array}{c}\text { Variable nominal } \\
\text { binaria: } \\
0=\text { Otras } \\
1=\text { Primera o } \\
\text { segunda opción }\end{array}$ \\
\hline Grado & Independientes & Carrera de grado que cursa & Grado & $\begin{array}{c}\text { Variable nominal: } \\
1=\text { ICA } \\
2=\text { IA } \\
3=\text { IAM } \\
\text { 4=BIOECO } \\
5=\text { IND } \\
6=\text { IAGRI17 } \\
7=\text { IAGRI10 }\end{array}$ \\
\hline Curso & & Curso (por exceso) que está realizando & Curso & $\begin{aligned} \text { Variable ordinal: } & \begin{aligned} 1 & =1 \\
2 & =2 \\
3 & =3 \\
4 & =4\end{aligned}\end{aligned}$ \\
\hline Edad & & $\begin{array}{c}\text { Edad en el momento en el que se realizó la } \\
\text { encuesta }\end{array}$ & Edad & Variable continua \\
\hline Género & & Sexo & Sexo & $\begin{array}{c}\text { Variable nominal } \\
\text { binaria: } \\
\text { 1=Hombre } \\
2=\text { Mujer }\end{array}$ \\
\hline Provincia & & $\begin{array}{c}\text { Si pertenece o no a la Comunidad de } \\
\text { Madrid }\end{array}$ & Provincia & $\begin{array}{c}\text { Variable nominal } \\
\text { binaria: } \\
1=\mathrm{SI} \\
0=\text { No }\end{array}$ \\
\hline Centro & & $\begin{array}{l}\text { Titularidad del centro educativo de } \\
\text { procedencia previo a la ETSIAAB }\end{array}$ & Centro & $\begin{array}{l}\text { Variable nominal: } \\
\text { 1=Público } \\
\text { 2=Privado } \\
\text { 3=Concertado } \\
\text { 4=Fuera de } \\
\text { España }\end{array}$ \\
\hline
\end{tabular}

Fuente: Elaboración propia 
Tabla 2 (Continuación)

Definición de variables

\begin{tabular}{|c|c|c|c|c|}
\hline Variable & $\begin{array}{l}\text { Tipo de } \\
\text { Variable }\end{array}$ & Definición & Nombre & Valores \\
\hline Especialidad & & $\begin{array}{c}\text { Modalidad cursada en el } \\
\text { Bachillerato }\end{array}$ & Especialidad & 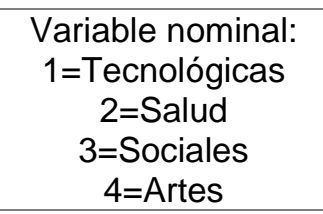 \\
\hline $\begin{array}{l}\text { Momento de la } \\
\text { elección del grado }\end{array}$ & & $\begin{array}{l}\text { Momento temporal cuando } \\
\text { tomó la decisión del grado que } \\
\text { quería cursar }\end{array}$ & Momento & $\begin{array}{c}\text { Variable nominal: } \\
\text { 1=Antes del } \\
\text { Bachillerato } \\
\text { 2=Después de la } \\
\text { EBAU } \\
\text { 3=Durante el } \\
\text { Bachillerato } \\
\text { 4=Entre el } \\
\text { Bachillerato y la } \\
\text { EBAU }\end{array}$ \\
\hline $\begin{array}{l}\text { Motivo de la } \\
\text { elección del grado }\end{array}$ & & $\begin{array}{l}\text { Motivación principal para tomar } \\
\text { la decisión del grado a cursar. }\end{array}$ & Motivo & $\begin{array}{c}\text { Variable nominal: } \\
\text { 1=Vocacional. } \\
2=\text { Afinidad o gusto } \\
\text { 3=Tradición familiar. } \\
\text { 4=Por descarte. }\end{array}$ \\
\hline Cambio de grado & & $\begin{array}{l}\text { Si pretende cambiarse de } \\
\text { grado en el próximo curso }\end{array}$ & Cambio & $\begin{array}{c}0=\text { No } \\
1=\text { Sí }\end{array}$ \\
\hline
\end{tabular}

Fuente: Elaboración propia

\section{Metodología}

En cuanto a la metodología, se han utilizado técnicas propias de la estadística descriptiva, así como un análisis de frecuencias. Posteriormente, se ha realizado un contraste estadístico, con el test de la Chi-cuadrado de Pearson por pares de variables, para analizar la significación existente entre aquellas más importantes, con el fin de conseguir los objetivos propuestos. Por último, se ha realizado un análisis factorial, una técnica estadística contrastada para poder alcanzar los propósitos que se plantean en este trabajo (Hair, Anderson, Tatham y Black, 2004). El análisis factorial exploratorio es considerado una importante herramienta en el estudio de ciencias sociales y es un modelo habitualmente utilizado en investigaciones educativas que nos sirve para identificar y agrupar los principales factores y componentes de los mismos que colaboran en la sucesión de un hecho (Gámez y Marrero, 2003; Caldera et al., 2018; Rodríguez-Muñiz et al. 2019; Sánchez-Almeida et al., 2020). 


\section{Resultados}

Análisis descriptivo, de frecuencias y de correlaciones

A continuación, se muestran los resultados del análisis descriptivo (tabla 3). Los datos de las frecuencias de la muestra en general -variables más importantes- y sus correlaciones se exponen y presentan en el texto posterior, en la discusión de los resultados, para que sea más sencilla su lectura simplificada y posterior comprensión.

\section{Tabla 3}

Estadísticos descriptivos

\begin{tabular}{lcccc}
\hline Variable & Mínimo & Máximo & Media & Desv. típ. \\
\hline Prioridad & 0 & 1 & 0,81 & 0,395 \\
\hline Curso & 1 & 4 & 2,36 & 1,098 \\
\hline Grado & 1 & 7 & 3,50 & 2,102 \\
\hline Edad & 17 & 47 & 21,23 & 3,251 \\
\hline Género & 1 & 2 & 1,51 & 0,500 \\
\hline Provincia & 0 & 1 & 0,77 & 0,420 \\
\hline Centro & 1 & 4 & 1,68 & 0,851 \\
\hline Especialidad & 0 & 4 & 1,61 & 0,559 \\
\hline Momento & 0 & 4 & 2,54 & 1,004 \\
\hline Motivo & 1 & 4 & 2,14 & 1,018 \\
\hline Cambio & 0 & 1 & 0,07 & 0,259 \\
\hline
\end{tabular}

Fuente: Elaboración propia

\section{Análisis factorial}

Se ha sometido a las variables al test de esfericidad de Barlett (1950); consiste en una estimación de Chi-cuadrado a partir de una transformación del determinante de la matriz de correlaciones. El nivel de significatividad es inferior a 0,05 por lo que se rechaza $\mathrm{H} 0$ de esfericidad, de manera que el modelo es adecuado para explicar los datos. A continuación, se considera el índice KaiserMeyer-Olkin (KMO), que compara las magnitudes de los coeficientes de correlación simple con las magnitudes de los coeficientes de correlación parcial. Valores cercanos a 0,5 indican que es adecuado utilizar el análisis factorial; no es el mejor valor, puesto que es mejor que sean valores superiores a 0,5 pero dado que el test de Barlett sale aceptable y el KMO es cercano a 0,5 se ha decidido aplicar este método. La experiencia práctica aconseja que es precipitado tomar el índice KMO como única medida de adecuación de la muestra a las hipótesis del modelo de análisis factorial, sobre todo si hay un número pequeño de variables consideradas. La tabla 4 muestra la prueba de Barlett y el índice KMO. 


\section{Tabla 4}

Tabla KMO y prueba de Bartlett(a)

Medida de adecuación muestral de Kaiser-Meyer-Olkin

0,467

\begin{tabular}{|c|c|c|}
\hline \multirow{3}{*}{ Prueba de esfericidad de Bartlett } & Chi-cuadrado aproximado & 93,195 \\
\hline & Gl & 36 \\
\hline & Sig. & 0,000 \\
\hline
\end{tabular}

a Sólo aquellos casos para los que PRI_BINARIA = primera o segunda opción, serán utilizados en la fase de análisis.

Fuente: Elaboración propia a partir de los resultados obtenidos en el SPSS

Una vez observada la adecuación para la realización del análisis factorial exploratorio a este conjunto de variables, se procede a llevar a cabo la siguiente fase que consiste en la extracción de componentes, a través de la agrupación de las 9 variables originales en unas nuevas (llamadas componentes o factores), las cuales son combinaciones de las originales. Para ello, se ha utilizado el método de componentes principales, como método de extracción de los mismos, en el que los factores obtenidos son autovectores de la matriz de correlaciones re-escalados. Se ha optado por establecer una determinación a priori, como puede observarse en la tabla 5. Las cargas indican el grado de correspondencia entre la variable y el factor, es decir, que cargas altas indican que dicha variable es representativa para dicho factor. Puede observarse que en el componente uno tiene bastante representatividad la provincia de residencia; en el componente dos, la edad; en el componente tres el cambio de titulación, y en el cuatro, el motivo de la elección. La tabla 5 recoge los datos obtenidos para los cuatro primeros componentes.

\section{Tabla 5}

Matriz de componentes ( $a, b)$

\begin{tabular}{ccccc}
\hline & \multicolumn{4}{c}{ Componente } \\
\cline { 2 - 5 } & 1 & 2 & 3 & 4 \\
\hline Grado & $-0,374$ & 0,160 & 0,152 & $-0,364$ \\
\hline Edad & 0,073 & $\mathbf{0 , 6 3 8}$ & $-0,389$ & 0,360 \\
\hline Género & 0,477 & $-0,363$ & $-0,305$ & 0,073 \\
\hline Provincia & $\mathbf{0 , 7 0 6}$ & 0,321 & 0,190 & $-0,001$ \\
\hline Centro & 0,500 & 0,404 & 0,277 & $-0,287$ \\
\hline Especialidad & 0,454 & $-0,462$ & 0,073 & $-0,410$ \\
\hline Momento & 0,152 & $-0,387$ & $-0,104$ & 0,522 \\
\hline Motivo & 0,227 & 0,014 & 0,469 & $\mathbf{0 , 4 6 1}$ \\
\hline Cambio & $-0,227$ & $-0,100$ & $\mathbf{0 , 7 2 9}$ & 0,227
\end{tabular}

Método de extracción: Análisis de componentes principales.

a 4 componentes extraídos

b Sólo aquellos casos para los que PRI BINARIA = primera o segunda opción, serán utilizados en la fase de análisis.

Fuente: Elaboración propia a partir de los resultados obtenidos en el SPSS

La figura 1 recoge el gráfico de sedimentación, que muestra una representación gráfica de la magnitud de los autovalores. El corte en la tendencia descendente sirve de regla para la determinación del número óptimo de valores que deben estar presentes en la elección. Como se puede observar, en principio parece factible la extracción de cuatro factores. 


\section{Figura 1}

Gráfico de sedimentación

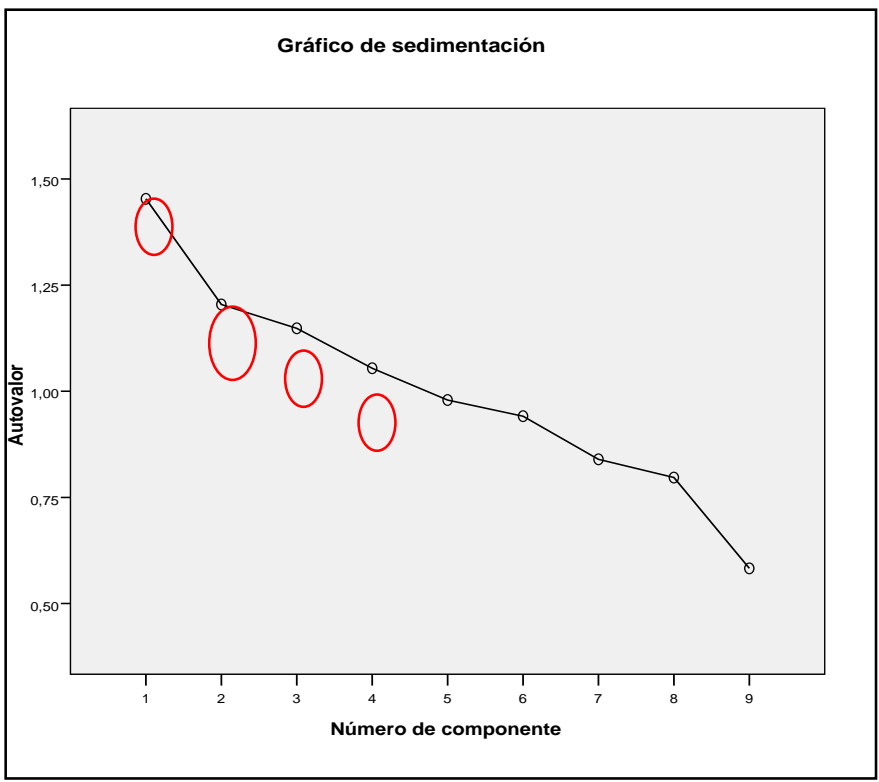

Fuente: Elaboración propia

La tabla 6 expone los datos de varianza total explicada. Como se puede observar, únicamente los cuatro primeros factores tienen valores propios mayores que 1 y explican el $54,002 \%$ de la varianza. Esto significa que con estos cuatro factores se puede representar un $54,002 \%$ del problema original. Así son relevantes estos cuatro factores para resumir las variables originales de la investigación que se plantea.

\section{Tabla 6}

Varianza total explicada(a)

\begin{tabular}{cccc}
\hline \multirow{3}{*}{ Componente } & \multicolumn{3}{c}{ Autovalores iniciales } \\
\cline { 2 - 4 } & Total & \% de la varianza & \% acumulado \\
\hline 1 & $\mathbf{1 , 4 5 3}$ & $\mathbf{1 6 , 1 4 8}$ & 16,148 \\
\hline 2 & $\mathbf{1 , 2 0 4}$ & $\mathbf{1 3 , 3 8 3}$ & $\mathbf{2 9 , 5 3 1}$ \\
\hline 3 & $\mathbf{1 , 1 4 8}$ & $\mathbf{1 2 , 7 5 8}$ & $\mathbf{4 2 , 2 8 9}$ \\
\hline 4 & $\mathbf{1 , 0 5 4}$ & $\mathbf{1 1 , 7 1 4}$ & 54,002 \\
\hline 5 &, 979 & 10,881 & 64,883 \\
\hline 6 &, 941 & 10,458 & $\mathbf{7 5 , 3 4 1}$ \\
\hline 7 &, 840 & 9,330 & 94,670 \\
\hline 8 &, 797 & 8,853 & 100,000 \\
\hline 9 &, 583 & 6,476 & \\
\hline
\end{tabular}

Método de extracción: Análisis de componentes principales.

a Sólo aquellos casos para los que PRI_BINARIA = primera o segunda opción, serán utilizados en la fase de análisis. Fuente: Elaboración propia a partir de los resultados obtenidos en el SPSS 
Resulta necesario en este punto efectuar una rotación ortogonal que permitirá reducir ambigüedades en las cargas factoriales de las variables y hallar una solución más clara. En la práctica, el objetivo de los métodos de rotación es simplificar filas o columnas de la matriz de factores para facilitar la interpretación. El método de rotación utilizado es VARIMAX que busca redistribuir la varianza a lo largo de todos los componentes en la matriz de carga. Con esto se simplifica el modelo y se obtienen resultados más claros para identificar los factores en cada componente, pues este método aproxima las cargas altas a $10-1$ y las cargas bajas de la matriz no rotada a 0 , eliminando de esta forma, las ambigüedades existentes en la matriz no rotada (Gámez y Marrero, 2003). Por último, en la tabla 7 se muestra la matriz de componentes rotados.

\section{Tabla 7}

Matriz de componentes rotados

\begin{tabular}{ccccc}
\hline \multirow{2}{*}{ Variables } & \multicolumn{4}{c}{ Componente } \\
\cline { 2 - 5 } & 1 & 2 & 3 & 4 \\
\hline Grado & $-0,097$ & $-0,556$ & 0,050 & $-0,011$ \\
\hline Edad & 0,197 & 0,108 & $-0,774$ & $-0,211$ \\
\hline Género & 0,097 & 0,567 & 0,241 & $-0,262$ \\
\hline Provincia & $\mathbf{0 , 7 7 3}$ & 0,192 & $-0,028$ & 0,058 \\
\hline Centro & $\mathbf{0 , 7 3 6}$ & $-0,167$ & 0,053 & 0,013 \\
\hline Especialidad & 0,235 & 0,175 & $\mathbf{0 , 6 9 3}$ & $-0,162$ \\
\hline Momento & $-0,214$ & $\mathbf{0 , 6 1 7}$ & $-0,006$ & 0,171 \\
\hline Motivo & 0,214 & 0,265 & $-0,084$ & $\mathbf{0 , 6 0 1}$ \\
\hline Cambio & $-0,082$ & $-0,166$ & 0,111 & $\mathbf{0 , 7 7 3}$ \\
\hline Fuente: Elaboración propia a partir de los resultados obtenidos en el SPSS & &
\end{tabular}

Podemos observar en esta matriz una clara agrupación de patrones donde prevalecen variables que definen los factores. De este modo hemos reducido las nueve variables originales a cuatro factores que representan cuatro bloques para el estudio de los factores que explican la elección de los estudiantes por los grados universitarios en el ámbito agroalimentario.

\section{Discusión}

Como puede observarse en la tabla 3, más del $80 \%$ de estudiantes eligieron su carrera en primera o segunda opción, y más del $70 \%$ eran de la misma ciudad donde cursaron sus estudios, resultados en línea con los trabajos de Rivas (1990), Mosteiro y Porto (2000) y Solsona et al. (2016).

El análisis de las frecuencias de la muestra general revela que a pesar de que la mayoría de los grados analizados son ingenierías, en el caso particular del área agroalimentaria hay paridad entre hombres (49\%) y mujeres $(51 \%)$ en el alumnado global. Estos resultados contradicen a autores como García Guevara (2002), Navarro y Casero (2012) y Doña y Luque (2019), que exponen que la elección de las mujeres suele encaminarse hacia carreras de humanidades, ciencias sociales, experimentales, jurídicas y relativas a la salud, mientras los hombres suelen decantarse por carreras de tipo técnico con el objetivo de alcanzar un buen salario. Así pues, parece ser que en las denominadas "titulaciones verdes", nombre a veces utilizado para designar a las carreras del sector agroalimentario, estas conclusiones no se aplican. 
Respecto a la frecuencia de la variable Centro, la mayoría de quienes eligieron los grados agroalimentarios en primera y segunda opción proceden de colegios públicos $(54,3 \%$ del total). El $25,3 \%$ y el $17,6 \%$ de los sujetos de la muestra proceden de centros privados y concertados, respectivamente, mientras que menos del $3 \%$ han estudiado fuera de España.

Por lo que se refiere al momento en que se tomó la decisión de qué grado cursar, la mayoría lo decidió justo antes $(22,3 \%)$ y después $(37,6 \%)$ de la prueba de la EBAU. Cabe destacar el pequeño porcentaje de estudiantes que tenía decidido qué grado cursar antes de empezar bachillerato (15\%); una de las decisiones más importantes que debe afrontar el alumnado de Educación Secundaria Obligatoria es sobre su itinerario educativo, lo que determina, no solo sus posibilidades de acceso a las diferentes titulaciones universitarias, sino también el éxito académico durante esta etapa (Corrales et al., 2020).

Por su parte, para casi la mitad de la muestra, la motivación principal para tomar la decisión del grado a cursar es la afinidad, y, en segundo lugar, por vocación. Así pues, en línea con los trabajos de Rivas (1990), Mosteiro y Porto (2000), Sánchez (2001) y Fernández et al. (2007), aproximadamente el $74 \%$ del alumnado eligió su carrera de forma vocacional o por afinidad. El $6,7 \%$ señala la tradición familiar como principal razón de su elección; en línea con Sánchez (2001) apunta que, para las carreras técnicas, como las que se presentan, la influencia socio familiar también es reseñable. Cabe destacar que casi un $20 \%$ eligieron por descarte.

Finalmente, el análisis de la frecuencia de la variable Cambio muestra que el $7,2 \%$ de las personas encuestadas tiene intención de abandonar la carrera elegida. Es muy probable que la mayoría de estas personas no eligieran el grado en primera o segunda opción, como puede comprobarse posteriormente en el análisis de correlaciones entre ambas variables. Este resultado está en consonancia con los de otros trabajos en los que se muestra una relación entre el rendimiento universitario y el grado de satisfacción con la titulación elegida (Moncada et al., 2019). Aquel estudiante que elige en el último momento, que no tiene claro qué titulación elegir o que se matricula en una titulación que no sea de su preferencia, es más probable que tenga problemas de rendimiento e insatisfacción (Álvarez-Pérez et al. 2015), e, incluso, está vinculado con el abandono de los estudios (Tuero et al., 2018).

En este apartado se analizan las correlaciones por pares de variables más importantes para conseguir alcanzar el objetivo planteado. En concreto, se analizará la existencia de una relación entre la variable objeto de explicación -la prioridad en la elección de los grados analizados- y las variables explicativas más relevantes (provincia, momento, motivo y cambio). Se omiten todas aquellas variables cuyos resultados no son significativos con el fin de hacer más sencillo y resumido el análisis.

El estudio de la correlación entre las variables prioridad-provincia muestra que 260 estudiantes que eligieron en primera o segunda opción el grado que están cursando en la actualidad pertenecen a la provincia de Madrid, frente a 87 que vienen de otra provincia. Por otro lado, de los 83 estudiantes que están cursando una titulación que no eligieron como primera o segunda opción, 72 residen en Madrid. Por lo que se refiere a la relación entre prioridad y momento, del total de estudiantes que están cursando una titulación que eligieron como primera o segunda opción, 56 tomó la decisión antes de empezar el bachillerato, 92 durante la realización de este, 79 lo decidió entre el bachillerato y la EBAU, y la mayoría (119 estudiantes) después de la EBAU. Respecto a la correlación entre las variables prioridad y motivo, de 347 estudiantes que están cursando el grado que eligieron en primera o segunda opción, 168 lo eligieron por afinidad, la decisión de 115 fue vocacional, 36 fue por descarte y los 28 restantes escogieron la titulación por tradición familiar. Finalmente, en cuanto a las variables prioridad-cambio, de 31 estudiantes afirma que pretende cambiarse de grado en el próximo curso, 17 que habían elegido otra carrera, frente a 14 que están cursando la titulación que habían elegido como primera o segunda opción.

A la vista de los resultados obtenidos, se puede concluir que existe una correlación significativa y positiva con la variable objeto de estudio, es decir, la decisión de cada estudiante de 
elegir el grado que está cursando en la actualidad como primera y segunda opción y con la ciudad de residencia (a mayor residencia en Madrid, mayor preferencia como primera o segunda opción de los grados objeto de análisis) en línea con trabajos como Rivas (1990), Mosteiro y Porto (2000) y Rego-Agraso y Rial-Sánchez (2017). El momento de decisión es importante también, existe una correlación positiva entre el momento de decisión y el hecho de elegir los grados en primera o segunda opción. En tercer lugar, se observa que el motivo de elección está directamente correlacionado con la elección de la carrera en primera o segunda opción. Así mismo, el abandono de la carrera está directamente relacionado con la elección de la carrera en primer o segunda opción (Tuero et al., 2018). Estos resultados están en línea con los obtenidos en otros trabajos revisados en la literatura, como Doña y Luque (2019) y Rodríguez-Muñiz et al. (2019).

Con respecto a los resultados obtenidos en el test de esfericidad de Barlett (1950), y al índice Kaiser-Meyer-Olkin (KMO) en la tabla 4, que compara las magnitudes de los coeficientes de correlación simple con las magnitudes de los coeficientes de correlación parcial. Se puede observar que su valor es superior a 0,4 , lo que indica que es adecuado utilizar el análisis factorial.

Una vez comprobada la adecuación para la realización del análisis factorial exploratorio a este conjunto de variables, en la tabla 5 , se ha procedido a hacer la extracción de componentes. Según el procedimiento de componentes principales se recomienda extraer 4 componentes, combinaciones de las 9 variables analizadas. Por otra parte, la figura 1, expuesta anteriormente, muestra el gráfico de sedimentación; como se puede observar, en principio parece factible la extracción de los cuatro factores citados.

Uno de los principales problemas que se plantea en este análisis es la elección del número de factores, si bien, se trata de una decisión que debe adoptar el propio investigador. En el presente trabajo se han elegido los cuatro primeros componentes, teniendo en cuenta que se pierde un $44 \%$ de la información original, siguiendo los márgenes aceptables para la extracción de componentes principales en este tipo de trabajos (Sánchez-Almeida et al., 2020). Las tablas 6 y 7 muestran los resultados obtenidos, los cuales evidencian, por tanto, la existencia de cuatro factores principales para la elección del grado en el ámbito agroalimentario, que son:

- Factor 1: incluye los datos de procedencia del estudiante, es decir, datos de residencia (si vivía o no en Madrid antes de acceder a la Universidad) y del tipo de centro de enseñanza de procedencia (si el centro educativo donde ha cursado bachillerato es privado, público, concertado o de fuera de España). Este factor explica el 16,14\% de la varianza total. En línea con los trabajos de Rivas (1990) y Mosteiro y Porto (2000).

- Factor 2: incluye el momento de la decisión en el que el estudiante elige el grado que quiere cursar (antes o durante el bachillerato, antes o después de la Ebau). Este factor explica el $13,38 \%$ de la varianza total.

- Factor 3: incluye como factor influyente la especialidad que el estudiante cursa en bachillerato (tecnológica, salud, sociales y artes) para explicar su elección de las titulaciones en primera y segunda opción. Este factor explica el $12,75 \%$ de la varianza total. En línea con los trabajos de Solsona et al. (2016) y Madden et al. (2018).

- Factor 4: incluye las motivaciones del estudiante para elegir la carrera (vocacional, tradición familiar, afinidad o descarte). Este factor explica el $11,71 \%$ de la varianza total. En línea con los trabajos de Sánchez (2001), Fernández et al. (2007), RegoAgraso y Rial-Sánchez (2017) y Doña y Luque (2019). 


\section{Conclusiones}

De forma general se pueden aceptar determinadas ideas compartidas sobre la relación existente entre la elección de las carreras universitarias y ciertos factores que influyen en dicha decisión. De hecho, quienes acceden a la universidad con suficiente información previa sobre las diferentes alternativas académicas, y además tienen claro cuáles son sus metas, estarán en mejor disposición para lograr una adecuada promoción en sus estudios y mejores resultados generales. Por el contrario, quienes eligen qué estudiar en el último momento, sin la información adecuada, que no tienen una idea clara sobre la titulación a elegir o que se matriculan en un grado que no sea de su preferencia, tendrán más probabilidades de sufrir problemas de bajo rendimiento e insatisfacción. Así, coincidiendo con la literatura previa (Domínguez et al., 2012; Álvarez-Pérez et al., 2015; Bernardo Gutiérrez et al. 2016; Álvarez-Pérez y López-Aguilar, 2019 y Rodríguez-Muñiz et al., 2019), se pone de manifiesto la importancia de una adecuada orientación educativa. Por su parte, otros trabajos muestran que existe una relación entre las asignaturas preferidas en la educación secundaria y la elección del grado universitario (Madden et al. 2018). De la misma forma, se asocia que el rendimiento universitario está directamente relacionado, entre otros factores, con el grado de satisfacción con la titulación elegida (Pablo-Lerchundi et al, 2014; Kinnunen et al., 2018).

A partir de estas conclusiones generales extraídas de la literatura, el presente trabajo ha pretendido contribuir al análisis para el caso concreto de las titulaciones de ámbito agroalimentario. Los resultados obtenidos para este ámbito concreto nos han permitido concluir que son cuatro los factores combinados más importantes: (1) la procedencia (lugar de residencia y tipología del centro escolar) del estudiante. En efecto, los estudiantes prefieren, en su mayoría, matricularse en universidades que estén cerca del lugar de la residencia familiar. (2) El momento de la decisión en el que el estudiante elige el grado que quiere cursar; la mayoría elige su carrera universitaria entre los meses de mayo-julio. (3) El itinerario académico previo (tecnológica, salud, sociales y artes) ya predispone a un tipo de carrera a elegir; y, por último, (4) sus motivaciones personales, como son las vocacionales, tradición familiar, afinidad o descarte.

Teniendo en cuenta estos factores, la orientación del alumnado en colegios e institutos es un elemento importante a tener en cuenta, sobre todo, para dar a conocer titulaciones que no son tendencia o moda en la actualidad. En concreto, consideramos que debe darse en las últimas etapas de formación más próximas al acceso a la universidad, haciendo partícipe a todos los agentes implicados en el proceso de toma de decisión del estudiante a la hora de elegir sus estudios superiores. Además, este asesoramiento debe estar adaptado a las necesidades de cada estudiante, en función de sus inquietudes, intereses y valores personales. Este trabajo pretende dar apoyo e información a los orientadores sobre los factores determinantes para la elección de un grado universitario, en concreto, un grado en el ámbito agroalimentario.

Coincidimos, por tanto, con otros trabajos previos (Álvarez-Pérez et al., 2015; Bernardo Gutiérrez et al. 2016; Álvarez-Pérez y López-Aguilar 2019; Rodríguez-Muñiz et al., 2019) que ponen de manifiesto la necesidad de mejorar la orientación que recibe el alumnado al respecto y, al mismo tiempo, señalan al docente preuniversitario como la persona adecuada en esta materia (Domínguez et al., 2012). Esta figura puede ofrecer información académica y asesorar sobre las diversas salidas profesionales a su alcance. De este modo, se incrementan las posibilidades de éxito y disminuyen las tasas de abandono universitario (Tuero et al., 2018). No debe perderse de vista que la educación superior es un pilar fundamental de desarrollo y bienestar, por lo que evitar el fracaso académico es un tema de especial relevancia. 


\section{Limitaciones y futuras líneas de investigación}

El trabajo tiene evidentes limitaciones técnicas; por un lado, se refiere a un momento concreto del tiempo, y no recoge datos de varios años que podrían ofrecer un análisis más profundo del tema. Por otro lado, la medición de las variables, la mayoría dicotómicas o categóricas, hace que se pierda mucha información explicativa en el modelo. También hay que tener en cuenta que el grupo de estudiantes que respondió la encuesta son quienes estaban en clase el día que se realizó. Esto incluye un sesgo en los resultados obtenidos pues se supone que quienes están en clase contestarán de forma más positiva que si hubieran contestado los que ya no asisten. En este sentido, si se hubiera hecho a quienes no acuden asiduamente, es probable que los resultados de la variable motivación fueran diferentes, al igual que otras variables.

Otra de las limitaciones del trabajo es su carácter local, ya que la encuesta se llevó a cabo en la Comunidad de Madrid, en la única Escuela que imparte este tipo de titulaciones de la región, la ETSIAAB de la UPM. En este sentido, sería interesante realizar una comparativa en otras Escuelas homólogas de provincias como la de Córdoba, Jaén, Lérida o León para ver qué resultados se obtienen. El carácter urbanita de la ciudad de Madrid puede condicionar el punto de vista de los estudiantes.

También, se recomienda completar el presente trabajo con variables de una medición cuantitativa, así como la inclusión de otras que completen el análisis como, por ejemplo, las que proponen Vázquez-Alonso y Manassero-Mas (2015): interés del alumnado por los temas científicos, la relación con profesores de ciencias, determinados programas de televisión y las expectativas futuras de empleo. Igualmente, sería interesante hacerlo en otros ámbitos como la química, física, matemáticas y otras áreas dentro de la ingeniería (industrial, telecomunicaciones, civil o informática), para poder comparar resultados y enriquecer las conclusiones sobre los factores que explican la elección de los estudiantes de las carreras universitarias, en particular de las carreras técnicas, ya que son las que han visto reducida su demanda en los últimos años.

Por último, sería interesante plantear un estudio con otro método que recoja datos de varios años, de tal forma que se puedan comparar relaciones entre variables consistentes o no en el tiempo. Esto puede ser especialmente interesante hoy en día, puesto que después de la crisis sanitaria, social y económica que estamos viviendo es posible que algunas ideas o paradigmas referentes a este tipo de profesiones y estudios puedan verse modificados.

\section{Referencias bibliográficas}

Álvarez-Pérez, P. R., López-Aguilar, D. y Pérez-Jorge, D. (2015). El alumnado universitario y la planificación de su proyecto formativo y profesional. Actualidades Investigativas en Educación, 15(1), 1-24. dx.doi.org/10.15517/aie.v15i1.16962.

Álvarez-Pérez, P. R. y López-Aguilar, D. (2019). Perfil de ingreso y problemas de adaptación del alumnado universitario según la perspectiva del profesorado. Revista Española de Orientación y Psicopedagogía, 30(3), 46-63. 
Bartlett, M. S. (1950). Tests of significance in factor analysis. British Journal of Statistical Psychology, 3(2), 77-85. https://doi.org/10.1111/j.2044-8317.1950.tb00285.x.

Bernardo, A., Esteban, M., Fernández, E., Cervero, A., Tuero, E. y Solano, P. (2016). Comparison of personal, social and academic variables related to university drop-out and persistence. Frontiers in Psychology, 7, 1-9. https://doi.org/10.3389/fpsyg.2016.01610.

Bethencourt Benítez, J. T., Cabrera Pérez, L., Hernández Cabrera, J. A., Álvarez Pérez, P. y González Afonso, M. (2008). Variables psicológicas y educativas en el abandono universitario. Revista Electrónica de Investigación Psicoeducativa, 6(3), 603-622. http://dx.doi.org/10.25115/ejrep.v6i16.129.

Boe, M. V. (2012). Science choices in Norwegian upper secondary school: What matters? Science Education, 96(1), 1-20. https://doi.org/10.1002/sce.20461.

Caldera Montes, J. F., Reynoso González, O. U., González Torres, Y.S. y Zamora Betancourt, M. R. (2018). Propiedades psicométricas de un instrumento de motivos de ingreso a carreras universitarias de ciencias de la salud. REDU. Revista de Docencia Universitaria, 16(1), 3350. https://doi.org/10.4995/redu.2018.6052.

Comisión Europea (2004). Increasing human resources for science and technology in Europe. Europe needs more scientists. Luxembourg: Office for Official Publications of the European Communities.

Corrales, M., Moreno, J., Sánchez, J. y Zamora, F. (2020). Estudio cualitativo de las motivaciones del alumnado de bachillerato en referencia a la modalidad de estudios. Campo Abierto. Revista de Educación, 39(1), 85-99. https://relatec.unex.es/revistas/index.php/campoabierto/article/view/3453.

Domínguez, G., Álvarez, F. J. y López, A. M. (2013). Acción tutorial y orientación en el periodo de transición de la educación secundaria a la universidad: La orientación al alumnado de nuevo ingreso. REDU: Revista de Docencia Universitaria, 11(2), 221-242.

Doña Toledo, L. y Luque Martínez, T. (2019). La experiencia universitaria. Análisis de factores motivacionales y sociodemográficos. Revista de la Educación Superior, 48(191), 1-24.

Fensham, P. J. (2004). Beyond Knowledge: Other Scientific Qualities as Outcomes for School Science Education. En R. M. Janiuk and E. Samonek-Miciuk (Ed.), Science and Technology Education for a Diverse World-dilemmas, needs and partnerships (23-25). Maria Curie-Sklodowska University Press.

Fernández, C., Peña, V., Viñuela, M. y Torío, S. (2007). Los procesos de orientación escolar y la toma de decisiones académica y profesional. Revista Complutense de Educación, 18(2), 87-103. http://hdl.handle.net/10651/48626.

Freixa, M., Llanes, J. y Venceslao, M. (2018). El abandono en el recorrido formativo del estudiante de ADE de la Universidad de Barcelona. Revista de Investigación Educativa, 36(1), 185202. https://doi.org/10.6018/rie.36.1.278971.

Gámez, E. y Marrero, H. (2003). Metas y motivos en la elección de la carrera universitaria: Un estudio comparativo entre psicología, derecho y biología. Anales de Psicología, 19(1), 121 131. https://revistas.um.es/analesps/article/view/27911.

García Guevara, P. (2002). Las carreras en ingeniería en el marco de la globalización: una perspectiva de género. Revista Latinoamericana en Estudios Educativos, 23(3), 91-105. https://www.redalyc.org/articulo.oa?id=270/27032305. 
González, I. (2005). Motivación y actitudes del alumnado universitario al inicio de la carrera. ¿Varían al egresar? Revista Electrónica de Investigación Psicoeducativa y Psicopedagógica, 5-3(1), 35-56.

Hair, J. F., Anderson R. E., Tatham R. L. y Black W. C. (2004). Análisis Multivariante. Pearson.

Kaiser, H. F. (1970). A second-generation little jiffy. Psychometrika, 35(4), 401-415. https://doi.org/10.1007/BF02291817.

Kinnunen, P., Butler, M., Morgan, M., Nylen, A., Peters, A. K., Sinclair, J., Kalvala, S. y Pesonen, E. (2018). Understanding initial undergraduate expectations and identity in computing studies. European Journal of Engineering Education, 43(2), 201-218. https://doi.org/10.1080/03043797.2016.1146233.

Madden, A. D., Webber, S., Ford, N. y Crowder, M. (2018). The relationship between students' subject preferences and their information behaviour. Journal of Documentation, 74(4), 692721. https://doi.org/10.1108/JD-07-2017-0097.

Martínez, A., Castro, M., Lucena, M. y Zurita, F. (2015). Elección de titulación universitaria y expectativas de resultados de los adolescentes de Granada. Revista Española de $\begin{array}{lllll}\text { Orientación } & y & \text { Psicopedagogía, } & \text { 63-77. }\end{array}$ https://www.redalyc.org/articulo.oa?id=3382/338245392004.

Martínez-Martínez, A., Zurita-Ortega, F., Castro-Sánchez, M., Espejo-Garcés, T., ChacónCuberos, R. y Ruiz-Rico-Ruiz, G. (2017). La elección de la lengua extranjera como titulación universitaria: Expectativas y rasgos de estudio. RELIEVE. Revista Electrónica de $\begin{array}{llll}\text { Investigación } y & \text { Evaluación } & \text { Educativa, } & \text { 23(2), }\end{array}$ https://doi.org/10.7203/relieve.23.2.8115.

Moncada, L., Negrete, F., Arias, M. y Armijos, R. (2019). Análisis de la triada: integración académica, permanencia y dispersión geográfica. RIED. Revista Iberoamericana de Educación a Distancia, 22(1). https://doi.org/10.5944/ried.22.1.22001.

Mosteiro García, M. y Porto Castro, A. (2000). Los motivos de elección de estudios en alumnos y alumnas de universidad. Innovación Educativa, 10, 121-132.

Navarro-Guzmán, C. y Casero-Martínez, A. (2012). Análisis de las diferencias de género en la elección de estudios universitarios. Estudios sobre Educación, 22, 115-132. https://hdl.handle.net/10171/22628.

Pablo-Lerchundi, I., Núñez-Del-Río, M. C. y González-Tirados, R. M. (2014). Career choice in engineering students: its relationship with motivation, satisfaction, and the development of professional plans. Annals of Psychology, 31(1), 268-279. https://doi.org/10.6018/analesps.31.1.159161.

Rego-Agraso, L. y Rial-Sánchez, A. (2017). ¿Por qué elegir formación profesional? Satisfacción, motivaciones y expectativas del alumnado sobre el empleo y la formación. Revista Española de Orientación y Psicopedagogía, 28(3), 43-62. https://www.redalyc.org/articulo.oa?id=3382/338254890002.

Rivas Martínez, F. (1990). La elección de estudios universitarios. Madrid: Consejo de Universidades, Secretaría General.

Rodríguez-Muñiz, L. J., Areces, D., Suárez-Álvarez, J., Cueli, M. y Muñiz, J. (2019). ¿Qué motivos tienen los estudiantes de Bachillerato para elegir una carrera universitaria? Revista de Psicología de la Educación, 14(1), 1-15. 
Sánchez, M. F. (2001). La orientación universitaria y las circunstancias de elección de los estudios. Revista de Investigación Educativa, 19(1), 39-61.

Sánchez-Almeida, T., Sandoval-Palis, I, Gilar-Corbi, R., Castejón-Costa, J. y Salazar-Orellana, D. (2020). Teaching evaluation questionnaire validation at Escuela Politécnica Nacional, applying the method of Factor Analysis with extraction of principal components. Ingeniería e Investigación, 40(1), 6-14. http://dx.doi.org/10.15446/ing.investig.v40n1.79634.

Solsona, J., Gómez, M. y Saladrigues, R. (2016). Análisis de los factores de elección de la Universidad de Lleida. Investigaciones de Economía de la Educación, 11(1), 77-89.

Tuero, E., Cervero, A., Esteban, M. y Bernardo, A. (2018). ¿Por qué abandonan los alumnos universitarios? Variables de influencia en el planteamiento y consolidación del abandono. Educación XX1, 21(2), 131-154. https://doi.org/10.5944/educXX1.20066.

Vázquez-Alonso, A. y Manassero-Mas, M. A. (2015). La elección de estudios superiores científicotécnicos. Revista Eureka sobre Enseñanza y Divulgación de las Ciencias, 12(2), 264-277. http://hdl.handle.net/10498/17251.

Vázquez Romero, I. M. y Blanco-Blanco, Á. (2019). Factores sociocognitivos asociados a la elección de estudios científico-matemáticos. Un análisis diferencial por sexo y curso en la Educación Secundaria. Revista de Investigación Educativa, 37(1), 269-286. http://dx.doi.org/10.6018/rie.37.1.303531.

Vieira, A., Monteiro, P., Carrieri, A., Guerra, V. y Brant, L. (2019). A study of relations between gender and careers anchors. Cadernos EBAPE. BR, 17(3), 577-589. https://doi.org/10.1590/1679-395172911x.

Fecha de entrada: 29 septiembre 2019

Fecha de revisión: 6 mayo 2020

Fecha de aceptación: 28 mayo 2020 